\section{New Commercial Products}

Announcements of new commercial products are published by the Journal of Applied Crystallography published by the Journal of Applied Crystallography
free of charge. The descriptions, up to 300 words or free of charge. The descriptions, up to 300 words or
the equivalent if a figure is included, should give the the equivalent if a figure is included, should give the
price and the manufacturer's full address. Full or partial inclusion is subject to the Editor's approval and to the space available. All correspondence should be sent to the Editor, $\operatorname{Dr} A$. M. Glazer, Editor Journal of Applied Crystallography, Clarendon Laboratory, University of Oxford, Parks Road, Oxford OX1 3PU, England.

The International Union of Crystallography can assume no responsibility for the accuracy of the claims made. A copy of the version sent to the printer is sent to the company concerned.

\section{J. Appl. Cryst. (1993). 26, 625}

\section{Radiation Alignment Device Shows}

\section{Precise Beam Location Safely}

A new safety alignment device for $\mathrm{X}$-rays, high-power lasers and other invisible radiation that lets users align experments safely by providing a narrow beam of red light is being introduced by Charles Supper Co., Inc. of Natick, Massachusetts.

SAFESIGHT is a compact device that provides users with a safe way to align devices that emit hazardous radiation. Simple to use, just place it in front of the radiation source, switch it on and a beam of safe red light from an integral laser

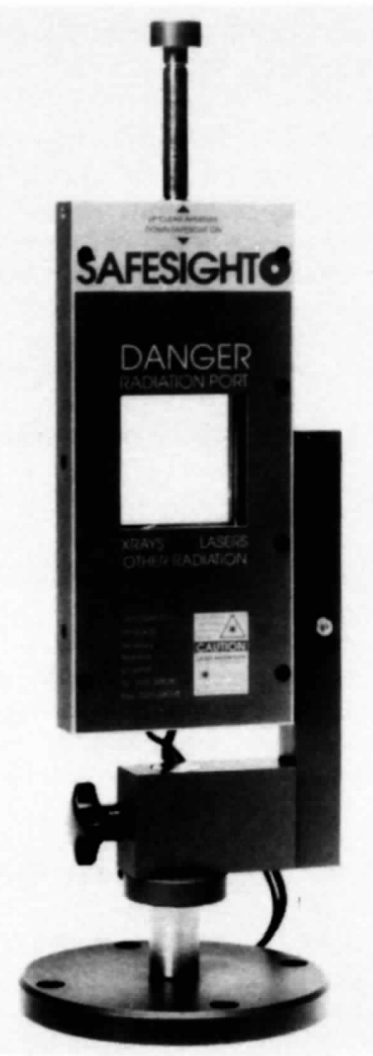

SAFESIGHT alignment device diode will be emitted from the center of the aperture in perfect alignment.

Once alignment is completed using the SAFESIGHT beam, then the aperture can be opened, allowing radiation to pass through without attenuation. To ensure maximum operator safety, the shutter can be interlocked with the radiation source. Apertures can range from 14 to $80 \mathrm{~mm}$ and options include mounts and remote controls.

SAFESIGHT is priced at $\$ 2495$ (list). Literature is available upon request.

Charles Supper Co., Inc., Donald E. Goodwin, Product Manager, 15 Tech Circle, Natick, MA 01760, USA

\section{Book Reviews}

Works intended for notice in this column should be sent direct to the Book-Review Editor (R. F. Bryan, Department of Chemistry, University of Virginia, McCormick Road, Charlottesville, Virginia 22901. USA). As far as practicable, books will be reviewed in a country different from that of publication.

\section{J. Appl. Cryst. (1993). 26, 625-626}

Inorganic materials. Edited by D. W. Bruce and D. O'Hare. Pp. xiv + 542. Chichester: John Wiley \& Sons, 1992. Price £55.00. ISBN 0471-92889-5.

This multi-author volume addresses a number of currently important aspects of materials chemistry. Topics covered in separate chapters include molecular inorganic superconductors, molecular inorganic magnetic materials, metalcontaining materials for nonlinear optics, inorganic intercalation compounds, biogenic inorganic materials, clay chemistry, polymeric coordination complexes, metal-containing liquid crystals and precursors for electronic materials.

The choice of authors for these chapters is definitely not a traditional one and features younger workers with European and British affiliations. This results in a refreshingly different point of view in many of the chapters dealing with areas most familiar to this reviewer. It would, however, have been helpful to have had the authors and their affiliations indicated in the table of contents, which looks misleadingly like that of a singleauthor volume.

The format of the chapters is in most cases similar, consisting of a reasonably detailed introduction to the basic principles relevant to a given area, followed by a review of work published in the last ten to twenty years. The inclusion of background material makes the text a very nice way of introducing the areas to a more general readership. The coverage is reasonably up-to-date, with only two chapters having no references prior to 1991 and several including references to work published in 1992. The breadth of coverage in the contributions varies substantially; some authors concentrate heavily on work from their own laboratory (e.g. molecular inorganic superconductors) while others provide a broader survey of established (e.g. intercalation chemistry) or developing (e.g. nonlinear optical materials) areas. The overall effect, however, is an approach that is distinctive without being idiosyncratic.

Thus, Chapter 1 , on molecular inorganic superconductors (P. Cassoux \& L. Valade, $58 \mathrm{pp}$.), is a case history of work in the authors' laboratories on metal-dmit complexes. The writing is perhaps overly dramatic for most tastes, with phrases such as 'the holy grail was found' and 'the fire of hope was ... kept burning', but the chapter gives a good review of the requirements for molecular superconductors. Chapter 2 (O. Kahn, Y. Pei \& Y. Journaux, $46 \mathrm{pp}$.) provides a detailed introduction to magnetic phenomena in solids and a good description of the correlation between structural and magnetic properties. After a thorough discussion of the physical principles underlying second- and third-order nonlinear optical phenomena, Chapter 3 (S. R. Marder, $50 \mathrm{pp}$.) provides a reasonably detailed review of the relevant properties of inorganic materials. The chapter on intercalation chemistry (Chapter 4, by D. O'Hare, $70 \mathrm{pp}$.) focuses on specifics without developing underlying principles; for example, oxidation of nitrogenous guests by metal dichalcogenides is probably a general phenomenon and is not restricted solely to the ammonia and pyridine systems cited.

Probably the most intriguing chapter is that on biogenic materials. (Chapter 5 , by $\mathrm{S}$. Mann, $57 \mathrm{pp}$.). It provides an excellent overview of this relatively unfamiliar area. The discussion of synthetic strategies to mimic such materials by using assembly in compartments or at monolayers, or molecules designed to interact specifically with a growing crystal surface, is compelling, despite the skepticism of some well regarded senior figures in the materials community. The chapters on clays (Chapter 6, by R. W. McCabe, 56 pp.) and on polymeric coordination complexes (Chapter 7, by G. E. Kellogg \& J. Gaudiello, 52 pp.) are much more in the nature of traditional reviews. In contrast, the chapter on metalcontaining liquid crystals (Chapter 8 , by D. W. Bruce, $86 \mathrm{pp}$.) provides a good introduction to liquid crystals in general, followed by a survey of methods used to incorporate metals. The final chapter 
(Chapter 9, by P. O'Brien, 44 pp.), on precursors to electronic materials, starts with a brief survey of electronic materials and growth methods and then focuses on IIIN and IIIVI systems, with a brief summary of methods applied to the high$T_{c}$ oxides. The book concludes with a very brief index that is too cursory to be useful.

Overall, the book is an asset to the literature. The production (from cameraready copy) is remarkably free of errors and the individual chapters are likely to be useful to relative newcomers as well as to experts in a given area. As an introduction to the excitement and promise of inorganic materials, it is clearly worth reading. The relative lack of emphasis on the detailed structures of the materials discussed, or on the methods used to elucidate them, may make the book of only peripheral interest to the practising crystallographer, but it provides a good introduction to new and potentially interesting areas of research.

BRUCE A. AVERILL

Department of Chemistry

University of Virginia

Charlottesville

VA 22901

USA

\section{Books Received}

J. Appl. Cryst. (1993). 26, 626

The following books have been received by the Editor. Brief and generally uncritical notices are given of works of marginal crystallographic interest; occasion ally, a book of fundamental interest is included under this heading because of difficulty in finding a suitable reviewer without great delay.

Crystallography in modern chemistry. A resource book of crystal structures.
By Thomas C. W. Mak and Gong-Du Zhou. Pp. xiii + 1323. New York: John Wiley, 1992. Price $\$ 136.00$. ISBN 0471-54702-6. A review of this book, by Jenny $P$. Glusker, has been published in the June 1993 issue of Acta Crystallographica Section B, pages 576-578.

Proton conductors - solids, membranes and gels - materials and devices. Edited by Phillipe Colomban. Pp. xxxii + 581. Cambridge: Cambridge University Press, 1992. Price £75.00. ISBN 0-521-38317-X. The stated aim of this book is to give a comprehensive survey of the chemical and physical parameters governing proton conduction. It includes descriptions of the preparation, structures and properties of typical materials (glasses, crystals, ceramics, metals, organic and inorganic polymers) and of devices'. 